\title{
BMJ Open Zinc for the prevention or treatment of acute viral respiratory tract infections in adults: a rapid systematic review and meta-analysis of randomised controlled trials
}

\author{
Jennifer Hunter (D) , ${ }^{1}$ Susan Arentz (D) , Joshua Goldenberg (D) , \\ Guoyan Yang (D) , Jennifer Beardsley (i) , ${ }^{3}$ Stephen P Myers (D) ,,4 \\ Dominik Mertz (iD , ${ }^{5}$ Stephen Leeder (iD ${ }^{6}$
}

To cite: Hunter J, Arentz S, Goldenberg J, et al. Zinc for the prevention or treatment of acute viral respiratory tract infections in adults: a rapid systematic review and meta-analysis of randomised controlled trials. BMJ Open 2021;11:e047474. doi:10.1136/ bmjopen-2020-047474

- Prepublication history and additional supplemental material for this paper are available online. To view these files, please visit the journal online (http://dx.doi.org/10.1136/ bmjopen-2020-047474).

Received 30 November 2020 Accepted 08 September 2021

D) Check for updates

(c) Author(s) (or their employer(s)) 2021. Re-use permitted under CC BY-NC. No commercial re-use. See rights and permissions. Published by BMJ.

For numbered affiliations see end of article.

Correspondence to Professor Jennifer Hunter; Jennifer.Hunter@ westernsydney.edu.au

\section{ABSTRACT}

Objective To evaluate the benefits and risks of zinc formulations compared with controls for prevention or treatment of acute viral respiratory tract infections (RTIs) in adults.

Method Seventeen English and Chinese databases were searched in April/May 2020 for randomised controlled trials (RCTs), and from April/May 2020 to August 2020 for SARS-CoV-2 RCTs. Cochrane rapid review methods were applied. Quality appraisals used the Risk of Bias 2.0 and Grading of Recommendations, Assessment, Development and Evaluation (GRADE) approach.

Results Twenty-eight RCTs with 5446 participants were identified. None were specific to SARS-CoV-2. Compared with placebo, oral or intranasal zinc prevented 5 RTIs per 100 person-months ( $95 \% \mathrm{Cl} 1$ to 8 , numbers needed to treat (NNT) $=20$, moderate-certainty/quality). Sublingual zinc did not prevent clinical colds following human rhinovirus inoculations (relative risk, RR $0.96,95 \% \mathrm{Cl}$ 0.77 to 1.21 , moderate-certainty/quality). On average, symptoms resolved 2 days earlier with sublingual or intranasal zinc compared with placebo $(95 \% \mathrm{Cl} 0.61$ to 3.50 , very low-certainty/quality) and 19 more adults per 100 were likely to remain symptomatic on day 7 without zinc (95\% Cl 2 to 38 , NNT=5, low-certainty/quality). There were clinically significant reductions in day 3 symptom severity scores (mean difference, MD -1.20 points, $95 \%$ $\mathrm{Cl}-0.66$ to -1.74 , low-certainty/quality), but not average daily symptom severity scores (standardised MD -0.15 , $95 \% \mathrm{Cl}-0.43$ to 0.13 , low-certainty/quality). Non-serious adverse events (AEs) (eg, nausea, mouth/nasal irritation) were higher (RR 1.41, 95\% Cl 1.17 to 1.69, NNHarm=7, moderate-certainty/quality). Compared with active controls, there were no differences in illness duration or AEs (low-certainty/quality). No serious AEs were reported in the 25 RCTs that monitored them (low-certainty/quality). Conclusions In adult populations unlikely to be zinc deficient, there was some evidence suggesting zinc might prevent RTIs symptoms and shorten duration. Non-serious AEs may limit tolerability for some. The comparative efficacy/effectiveness of different zinc formulations and doses were unclear. The GRADE-certainty/quality of the

\section{STRENGTHS AND LIMITATIONS OF THIS STUDY}

$\Rightarrow$ An extensive database search was conducted with no limits on language or date.

$\Rightarrow$ It is the first systematic review to analyse hazard ratios for symptomatic duration, day 3 mean symptom severity scores around the peak of acute respiratory illness and risks of adverse events.

$\Rightarrow$ The Risk of Bias 2.0 tool was used to appraise the risk of bias at the outcome level, and the Grading of Recommendations, Assessment, Development and Evaluation approach was used to appraise the quality/certainty of the evidence.

$\Rightarrow$ The study was limited by the rapid review methods, for example, where calibrated single reviewers were used.

$\Rightarrow$ Protocol changes and post hoc decisions as declared increased the risk of selective reporting bias.

evidence was limited by a high risk of bias, small sample sizes and/or heterogeneity. Further research, including SARS-CoV-2 clinical trials is warranted.

PROSPERO registration number CRD42020182044.

\section{BACKGROUND}

Acute viral respiratory tract infections (RTIs) are ubiquitous in the community. Clinical presentations range from milder cold and influenza-like illnesses to more serious conditions such as viral pneumonia and severe acute respiratory syndrome. Infection rates vary according to viral pathogen, location, season and the host's health status and age. ${ }^{1}$ Although most infections are self-limiting, the high incidence leads to substantial healthcare costs and broader economic impacts from school and work absenteeism. ${ }^{2}$

Except for influenza and SARS-CoV-2 vaccinations, prophylactic and therapeutic options are limited. Clinical practice guidelines focus 
on hand hygiene, reducing inappropriate antibiotic use and symptomatic relief with over-the-counter medications. ${ }^{3-5}$ Some guidelines recommend zinc. ${ }^{5}$ However, systematic reviews of zinc are limited by variations in administration route or formulation, are outdated, have been withdrawn or are low quality. ${ }^{6-11}$ The mechanisms for how zinc might work include broad spectrum antiviral properties in vitro against most of the common respiratory viruses, including coronaviruses. ${ }^{12-14}$ Zinc is important for immunity, inflammation, haemostasis, ACE 2 activity and also assists with tissue responses to hypoxia. ${ }^{131516}$ Not surprisingly then, zinc has garnered attention during the global COVID-19 pandemic. ${ }^{1315}$ Both high-income and low- income countries have seen increased zinc supplement use and sales. ${ }^{18}{ }^{19}$ Some healthcare workers, clinicians and hospitals are already using zinc to prevent or treat SARS-CoV-2 infections. ${ }^{20-31}$

In response to calls for rapid evidence appraisals to inform self-care and clinical practice during the COVID-19 pandemic, ${ }^{32}$ we developed a rapid systematic review protocol to evaluate zinc for the prevention and treatment of SARS-CoV-2 and other viral RTIs. ${ }^{33}{ }^{34}$ At the time of this review, results from COVID-19 randomised controlled trials (RCTs) were all pending. Therefore, this rapid review updates previous systematic reviews of RCTs investigating any type of zinc intervention to prevent or treat viral RTIs in adult populations.

\section{METHODS \\ Protocol}

This rapid review conforms with Cochrane guidance ${ }^{35} 36$ and the Preferred Reporting Items for Systematic Reviews and Meta-Analyses (PRISMA) (online supplemental file 1: PRISMA 2020 checklist). ${ }^{37}$ Following feedback from our content experts, who at that stage were blinded to the search results, amendments to the registered protocol ${ }^{33}$ were made pre data extraction and a revised protocol published. ${ }^{34}$ Postprotocol input from consumer/patient advocate representatives who were blinded to the results, led to minor changes to the rating of the importance of outcomes. Due to very serious concerns with the indirectness of the available evidence and the importance of not overstating its relevance to the pandemic, the post hoc decision was made to remove COVID-19 from the title and not Grading of Recommendations, Assessment, Development and Evaluation (GRADE) the certainty of the evidence in the context of SARS-CoV-2 prevention or treatment. Further details about amendments to the protocol and post hoc decisions are reported below.

\section{Search strategy}

A research librarian (JB) experienced with systematic review led the search (online supplemental appendix 1). PubMed, Embase, Cochrane CENTRAL, Academic Search Complete, Allied and Complementary Medicine Database, Alt Health Watch, CINAHL Plus with Full Text, Health Source, PsycINFO, China Knowledge Resource
Integrated Database (CNKI), U.S. National Library of Medicine Register of Clinical Trials (ClinicalTrials. gov), International Standard Randomized Controlled Trial Number Register (ISRCTN), World Health Organization International Clinical Trials Registry Platform (WHO ICTRP), Global Coronavirus COVID-19 Clinical Trial Tracker and Chinese Clinical Trial Registry were searched from inception up to 8 May 2020, with no limit on language. This was supplemented by bibliography searches of included articles, and due to no eligible RCTs being identified in the first search additional post hoc COVID-19 focused searches were conducted up to 19 August 2020 that included the addition of covid19-trials. org, and medRxiv and bioRxiv preprint databases.

\section{Eligibility criteria}

Study design

Included were randomised and quasi-randomised controlled trials. Excluded were systematic reviews, nonrandomised studies of interventions and studies without a concurrent control.

\section{Population}

Adults in any setting were included if they were at risk of contracting a viral RTI, had clinical illness with a laboratory confirmed viral RTI, or a non-specific respiratory tract illness that is predominantly caused by a viral infection, such as the common cold, non-seasonal rhinosinusitis, pharyngitis, laryngitis, influenza-like illness and healthy adults with acute bronchitis. Excluded were adults with bacterial infections and other respiratory illnesses when a viral infection was not confirmed.

\section{Interventions and comparators}

Included were interventions of any zinc conjugates, dose, duration and administration route. Excluded were co-interventions, including other nutraceuticals, herbs or pharmaceuticals unless both the intervention and control groups received the co-intervention. All types of controls and comparator groups were included.

\section{Outcomes}

A detailed list of critical (primary) and important (secondary) outcomes can be found in online supplemental appendix 2. Critical outcomes included incidence of RTIs, symptomatic survival, composite symptom severity scores, health-related quality of life (QoL) and serious and non-serious adverse events (AE). Important outcomes included the duration of symptoms and the number of different types of AEs.

\section{Data collection and appraisal}

In line with rapid review methods ${ }^{35}$ the first 30 title abstracts and 5 full papers were jointly screened for calibration. After which, single reviewers screened articles and a second reviewer screened the excluded articles (online supplemental appendix 2). Similarly, following calibration, single reviewers extracted data on the study design, funding, participants, interventions, comparators, 
outcomes measures and effect size and direction into a piloted electronic spreadsheet that was verified by a second reviewer. For articles published in Chinese the second reviewer used Google translate to verify data extraction. Single reviewers also appraised the risk of bias (RoB) of study outcomes with the Cochrane RoB 2.0 tool $^{38}$ that was verified by a second reviewer (online supplemental appendix 3). However, discrepancies in calibration led to the post hoc decision to apply recommended systematic review methods where two reviewers independently appraise the RoB. Any disagreements or uncertainties were discussed with the other reviewers and resolved through consensus. Other review constraints included only appraising the RoB of outcomes that were meta-analysed or the primary outcome, not imputing missing data for secondary outcomes and not contacting the authors. Instead, additional information from previous systematic reviews was extracted. ${ }^{7839}$ Data from graphical reports were extracted with WebPlotDigitizer V.4.2 (online supplemental appendix 4$){ }^{40}$

\section{Statistical methods and evidence synthesis}

RevMan V.5.4, ${ }^{41} \mathrm{R}$ software, ${ }^{42} 43$ Microsoft Excel and GRADEpro GDT ${ }^{44}$ were used. Studies reporting separate counts for different types of viral RTIs (eg, common cold, bronchitis, influenza-like illness) were combined to calculate the incidence of RTIs per person-months. Mean symptom severity scores were transformed to a modified Jackson common cold scale. ${ }^{45}{ }^{46}$ Means were used as a proxy for median days duration of symptoms. Data extracted from symptomatic survival curves was imputed for the first 7 days using the direct method 10 in the 'HR calculations spreadsheet' published by Tierney et al. ${ }^{47}$ Results and their $95 \%$ CIs are expressed as relative risks (RR) for dichotomous outcomes, incidence rate ratios (IRR) for person-time rates, mean difference (MD) or standardised MD (SMD) for continuous outcomes and hazard ratios (HR) for time-to-event outcomes. Absolute risks/rate differences and numbers needed to treat $(\mathrm{NNT})$ or harm $(\mathrm{NNH})$ are also reported. The MantelHaenszel method was used to calculate the pooled RR, generic inverse variance method was used for MD, SMD and IRR, and O-E variance method was used for HR. Irrespective of statistical heterogeneity, due to considerable clinical and methodological diversity/heterogeneity, random effects models were used.

The Cochran $Q$ test and I ${ }^{2}$ statistic were used to measure heterogeneity ${ }^{36}$ Subgroup analyses followed published methods and was assessed with the $\chi^{2}$ test. $^{48} \mathrm{~A}$ priori analyses compared age groups, RTI causes and severity, and zinc administration routes, salts and doses. Post hoc subgroup analyses compared days symptomatic prior to study enrolment and study definitions of symptomatic recovery. The three zinc dose subgroups $(<50 \mathrm{mg}$ daily, 50-200 mg daily, $>200 \mathrm{mg}$ daily of elemental zinc) were selected post hoc based on a no observed adverse effect level of $50 \mathrm{mg}$ and a higher risk of more severe AEs, such as vomiting, with doses above $225 \mathrm{mg} .^{49}$
For SMD the minimally important difference (MID) was set at $0.5 .^{50}$ Except for an MID of 1-day reduction in the duration of the common cold, ${ }^{51}$ there was little consensus in the literature on the MID for the other measures of effect; therefore, these were set post hoc. For symptom severity on day 3 for mild RTIs, the MID for MD was set at 1 point on a standardised scale that was the half-way mark between two proposed MIDs (online supplemental appendix 4). ${ }^{5152}$ Based on a $33 \%$ probability of remaining symptomatic on day 7 without any treatment, ${ }^{53}$ the MID for HRs was set at 1.9 (ie, NNT=5).

The GRADE approach was used to grade the certainty (quality) of the effects estimates and for the Summary of Findings table (online supplemental appendix 5). ${ }^{54}$ When data from at least 10 studies were pooled, funnel plots were created, visually inspected for publication bias and statistically analysed using Egger's regression for continuous outcomes and the Harbord score for dichotomous outcomes. However, due to ongoing methodological uncertainties, no statistical test was used for HRs. ${ }^{556}$

Throughout the manuscript, zinc doses are reported as milligrams (mg) of elemental zinc. Further details about protocol and post hoc changes, RoB appraisal, statistical methods and GRADE-certainty assessments can be found in online supplemental appendices 3-5.

\section{Patient and public involvement}

The protocol was rapidly developed in response to a call from the WHO for rapid evidence reviews to inform self-care and clinical practice during the COVID-19 pandemic. The World Naturopathic Federation responded by setting review topics. ${ }^{32}$ The zinc protocol was published prior to direct patient advocate involvement. Experienced Australian patient advocates have since provided input on the outcome measures and the presentation of the results and discussion.

\section{RESULTS}

From the 1360 articles and registered trials screened (online supplemental appendix 1), 28 unique RCTs, reported in 25 articles, with 5446 participants met the inclusion criteria (figure 1). $.^{51} 57-80$ Three were published in Chinese language only. ${ }^{77-79}$ Online supplemental appendix 2 lists the 95 RCTs evaluating zinc in paediatric populations, articles published in English that were excluded at full-paper screen, and the characteristics of the seven registered RCTs evaluating zinc for SARS-CoV-2, all with pending results.

\section{Study characteristics}

Study participants were generally healthy with clinical symptoms consistent with a mild to moderate viral RTI (online supplemental appendix 3). None were infected with the primary pathogen of interest SARSCoV-2. Only $1^{74}$ of the 20 RCTs ${ }^{5161-80}$ with communityacquired RTI reported the number of participants 


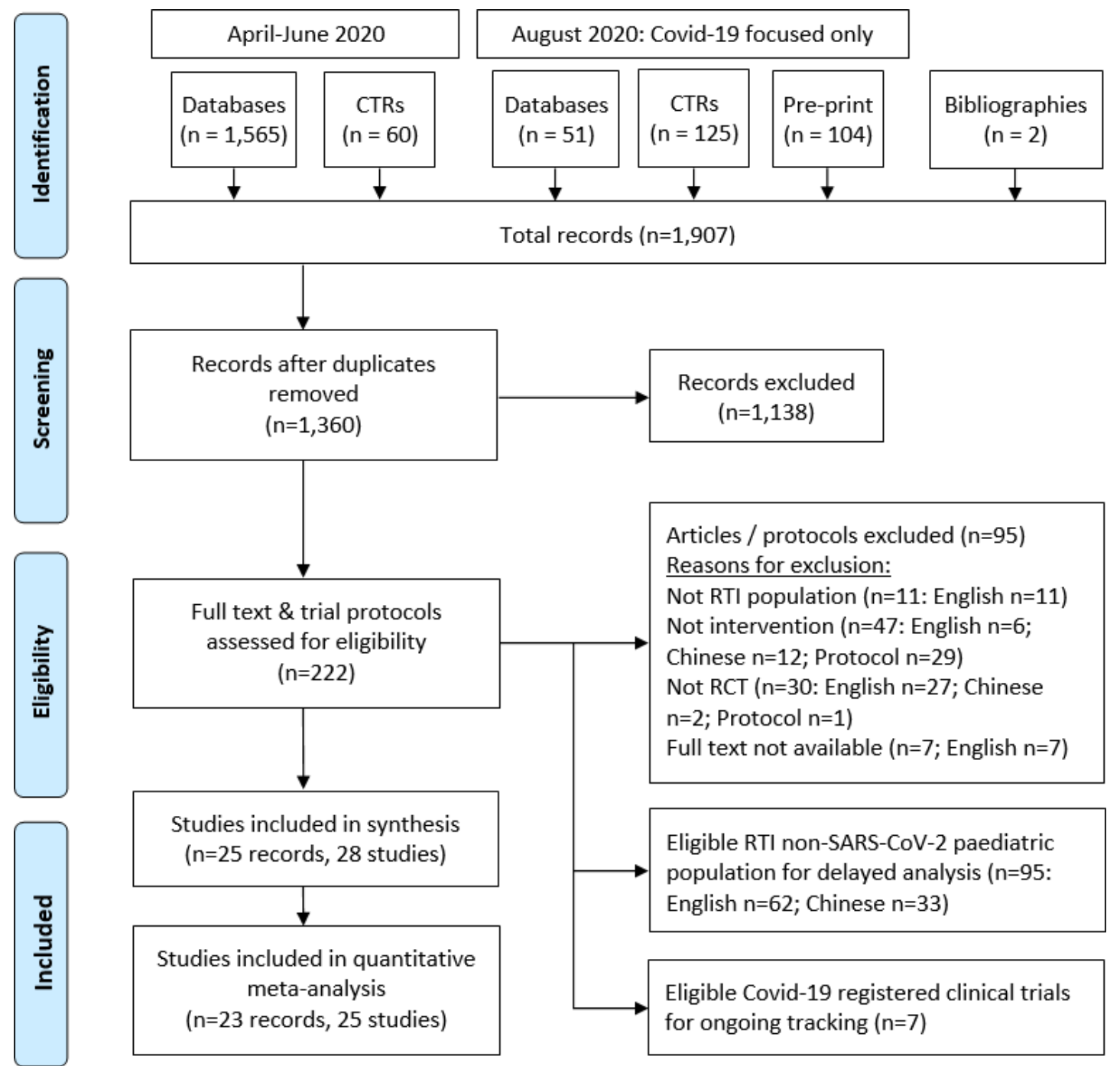

Figure 1 Search results flow chart. CTRs, clinical trial registries; RTIs, respiratory tract infections.

with a proven viral infection. Six RCTs inoculated participants with a human rhinovirus strain (HRV 2, 13, 23 or 39). ${ }^{51}{ }^{58-60}$ Most participants were younger than 65 years. Two RCTs also included older children and adolescents, notwithstanding, the mean age was around 37 years. ${ }^{66} 66$ Two RCTs included older adults from different ethnic backgrounds in the USA, many of whom had chronic disease comorbidities and were taking long-term medication. ${ }^{57} 76 \mathrm{In}$ another RCT, around one-third of participants had a history of asthma ${ }^{67}$ In the two RCTs that used oral zinc for prevention, zinc deficiency was excluded prior to enrolment. ${ }^{7576}$

Most studies were single centre, two-arm RCTs $(\mathrm{n}=26)^{57-78} 80$ and were conducted in the USA $(\mathrm{n}=19), 515759-666870-737576$ followed by western Europe $(\mathrm{n}=5),{ }^{58} 676980$ China $(\mathrm{n}=3)^{77-79}$ and Australia $(\mathrm{n}=1){ }^{74}$ The median sample size for prevention studies was 53 (range 32-1945) and for treatment studies 78 (range 12-279). At least half reported the RCT had sufficient statistical power for the study's primary outcome(s).
All but two RCTs reported at least one result that was used in a meta-analysis of a critical or important outcome ${ }^{5779}$ None of the RCTs reported mortality or other clinical outcomes relevant to severe or critical illness from acute viral RTIs or QoL outcomes. Four RCTs evaluated zinc for prevention, ${ }^{75-78}$ and 17 RCTs for treatment $t^{51-71-747980}$ of symptoms consistent with a community-acquired viral RTI. Of the six RCTs that inoculated participants with HRV, four RCTs evaluated zinc for both prevention and treatment, ${ }^{58-60}$ one RCT for treatment only ${ }^{51}$ and one assessed the tolerability and AEs of a zinc lozenge. ${ }^{58}$ Another RCT assessed AEs and safety of a zinc lozenge used by older adults. ${ }^{57}$

The most common zinc formulations were lozenges followed by nasal sprays and gels containing either zinc acetate or gluconate salts. The daily dose of prophylactic oral zinc for community-acquired infections was $15 \mathrm{mg}^{75}$ or $45 \mathrm{mg}^{76}$ for 7 or 12 months, respectively. Sublingual lozenge doses to prevent or treat HRV inoculation and community-acquired infections ranged between $45 \mathrm{mg}$ and $300 \mathrm{mg}$ daily and were used 
Community aquired mild to moderate RTIs (per person-months)

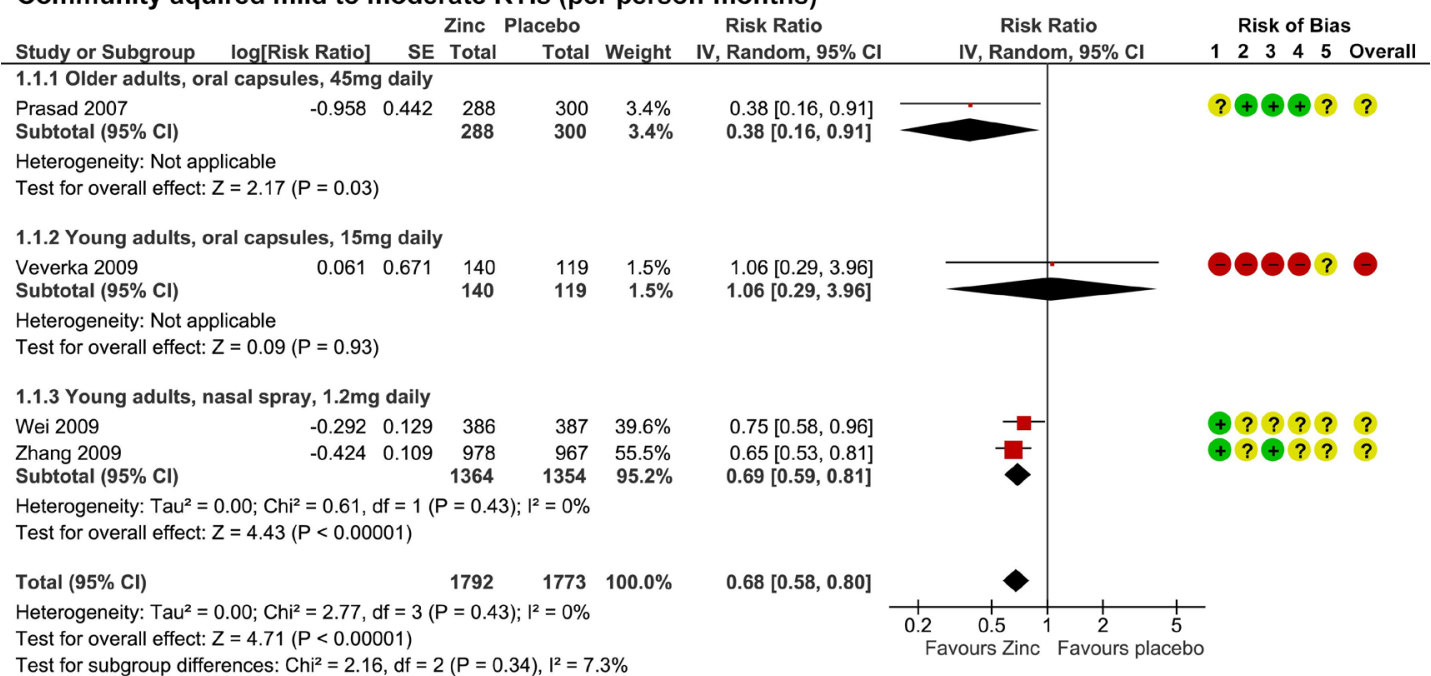

Test for subgroup differences: $\mathrm{Chi}^{2}=2.16, \mathrm{df}=2(\mathrm{P}=0.34), \mathrm{I}^{2}=7.3 \%$

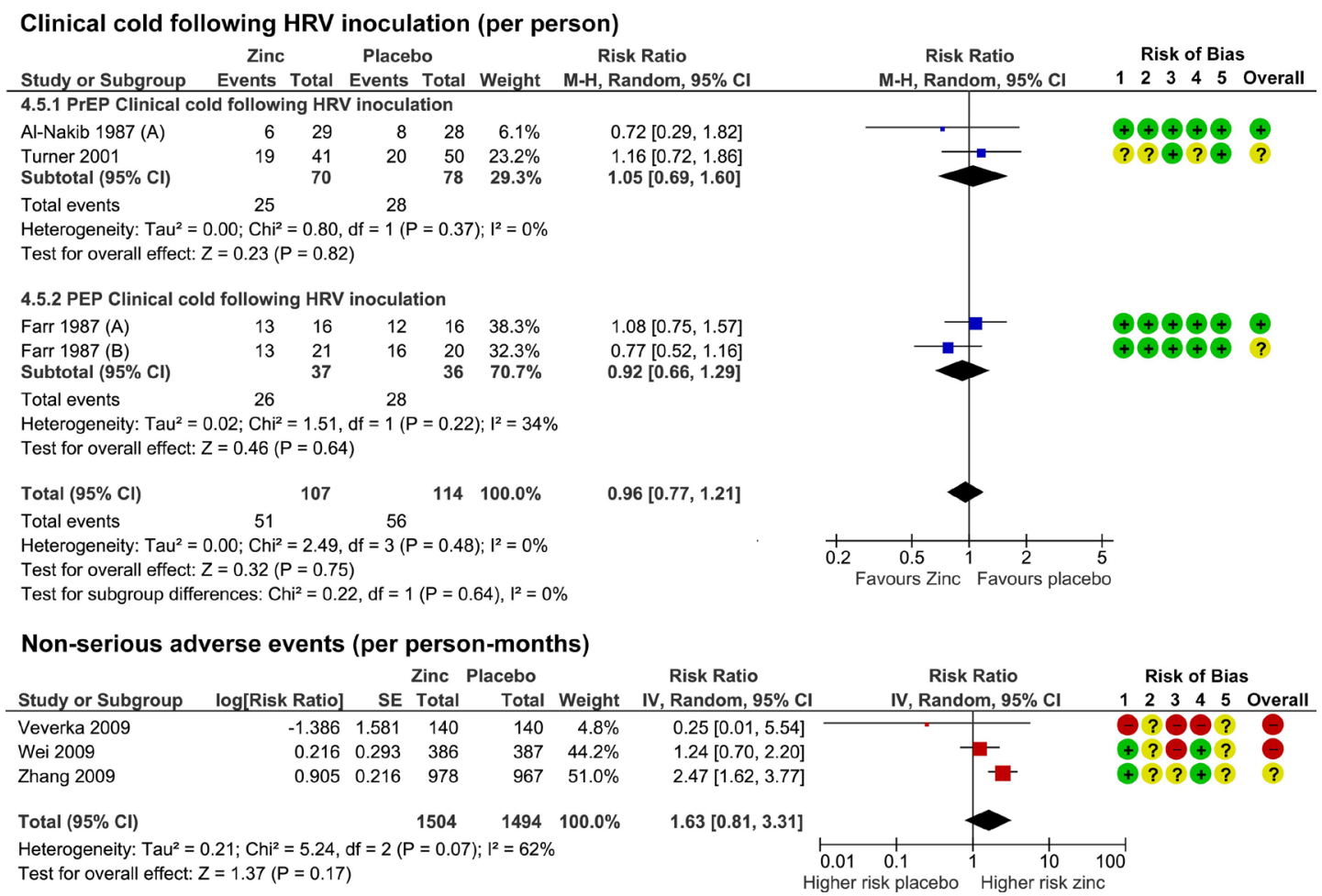

Figure 2 Prevention of respiratory tract infections (RTIs). Risk of community-acquired RTI, clinical colds from human rhinovirus (HRV) inoculation and non-serious adverse effects from prophylaxis. RoB-2 risk of bias legend: (1) randomisation process, (2) deviations from intended interventions, (3) missing outcome data, (4) measurement of the outcome, (5) selection of the reported result. M-H, Mantel-Haenszel; PEP, post-exposure prevention; PREP, pre-exposure prevention; RoB, risk of bias.

for up to 2 weeks. $^{5158-6163-676971-74}$ Doses for topical nasal zinc to prevent or treat community-acquired infections were substantially lower (0.9-2.6 mg/ day). ${ }^{62} 66687077-79$ Twenty-five RCTs compared zinc to a placebo that was matched or partially matched. Two 4-arm RCTs used an active control lozenge containing quinine hydrochloride ${ }^{51}$ and a 2-arm RCT used a nasal spray containing naphazoline hydrochloride. ${ }^{79}$

Certainty and quality of the evidence

Most of the prevention, duration, severity and $\mathrm{AE}$ outcomes had at least some concerns about their overall RoB (online supplemental appendix 3 and figures 2-5). Consequently, except for the prevention of RTIs following HRV inoculation ${ }^{58-60}$ and risk of non-serious AEs with zinc compared to an active control, ${ }^{6079}$ the GRADE certainty/quality of evidence was downgraded by one level for RoB (online supplemental appendix 5). This included the outcomes in which some studies had a high RoB, as the estimates of effects were robust following removal of these RCTs (online supplemental appendix 4). Serious AEs and symptom severity outcomes were downgraded another 
Day-3 symptom severity score

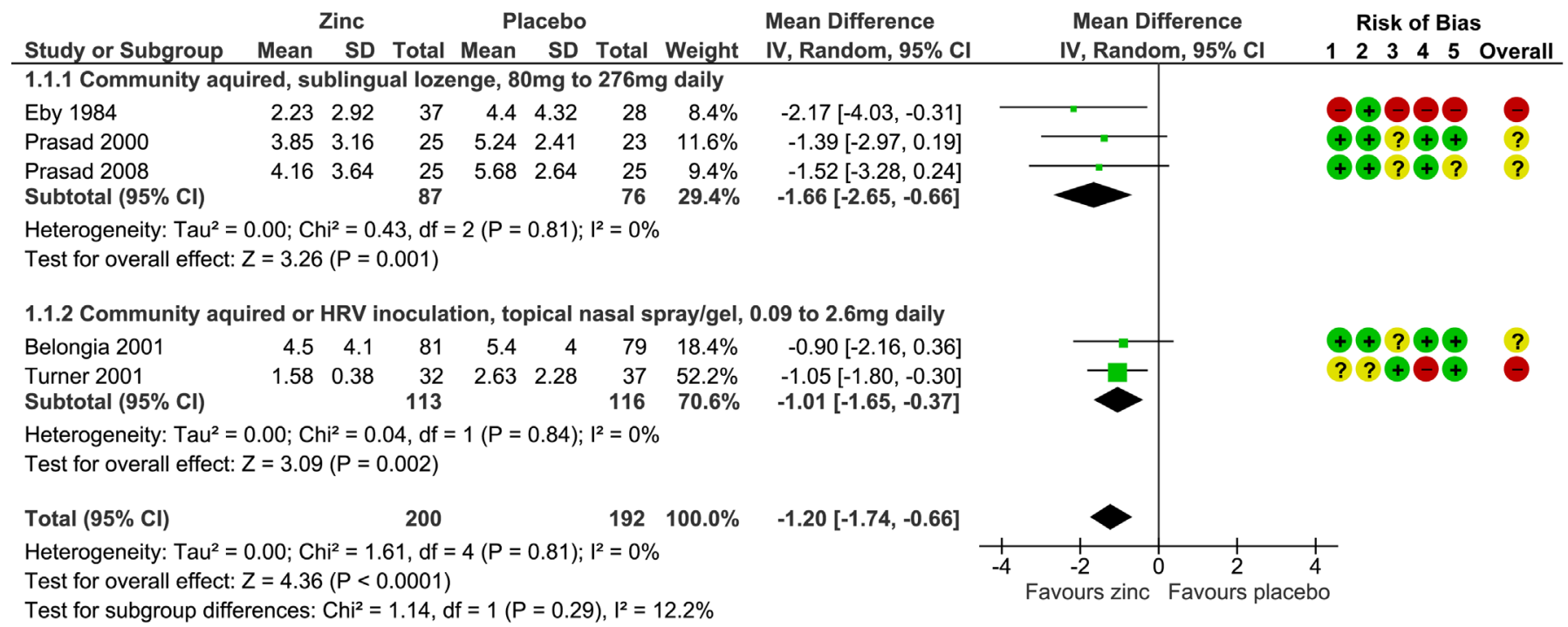

\section{Average daily symptom severity score}

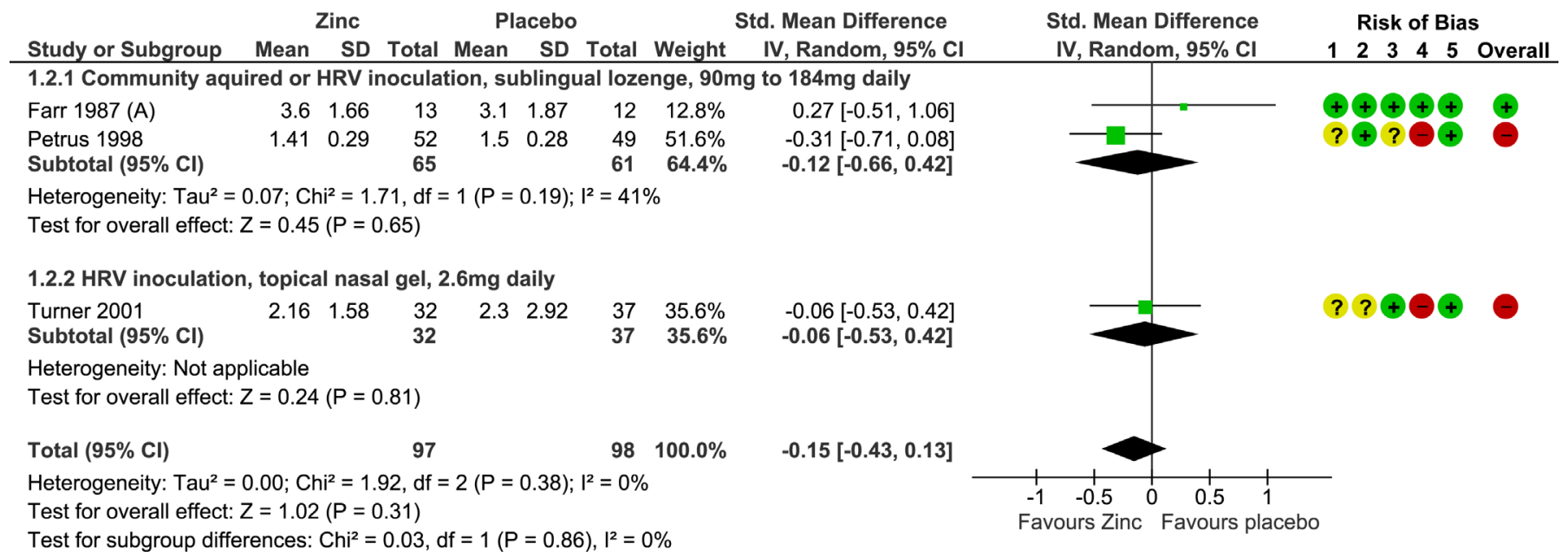

Figure 3 Symptom severity. Mean symptom severity scores following treatment for community-acquired respiratory tract infections and clinical colds from human rhinovirus (HRV) inoculation. RoB-2 risk of bias legend: (1) randomisation process, (2) deviations from intended interventions, (3) missing outcome data, (4) measurement of the outcome, (5) selection of the reported result. RoB, risk of bias.

level for imprecision due to the small pooled-sample sizes (online supplemental appendix 5). There was considerable statistical heterogeneity for the zinc versus placebo duration outcomes (figure 4). The HR effect estimate was downgraded one level for inconsistency, as the heterogeneity was partially explained by clinical and methodological diversity in the subgroup and sensitivity analyses (online supplemental appendix 4 ) and the $95 \%$ CI mostly overlapped (figure 3). However, the mean days duration was downgraded by two levels, due to conflicting evidence from clinically important positive and negative effects, minimal overlapping of the 95\% CI (figure 3) and neither the subgroup nor sensitivity analyses substantially reduced the heterogeneity (online supplemental appendix 4). At least 11 RCTs were industry funded, with a further seven receiving partial industry support (online supplemental appendix 3). Publication bias was not strongly suspected (online supplemental appendices $4,5)$.

\section{Findings from prevention studies}

Community-acquired infections

When oral or topical nasal zinc was compared with placebo controls, there was moderate certainty/quality evidence of a $32 \%$ lower RR of developing mild to moderate symptoms consistent with a viral RTI (IRR $0.68,95 \%$ CI 0.58 to 0.80 ) (figure 2) ${ }^{75-78}$ Five RTIs per 100 person-months of zinc use were prevented (95\% CI 1 to $8, \mathrm{NNT}=20$ ). The largest reductions in RR were for moderately severe symptoms consistent with an influenza-like illness (eg, elevated temperature). There was an $87 \%$ lower risk of developing moderately severe symptoms (IRR $0.13,95 \%$ 
Risk of remaining symptomatic over 7-days: sublingual or topical nasal zinc compared to placebo

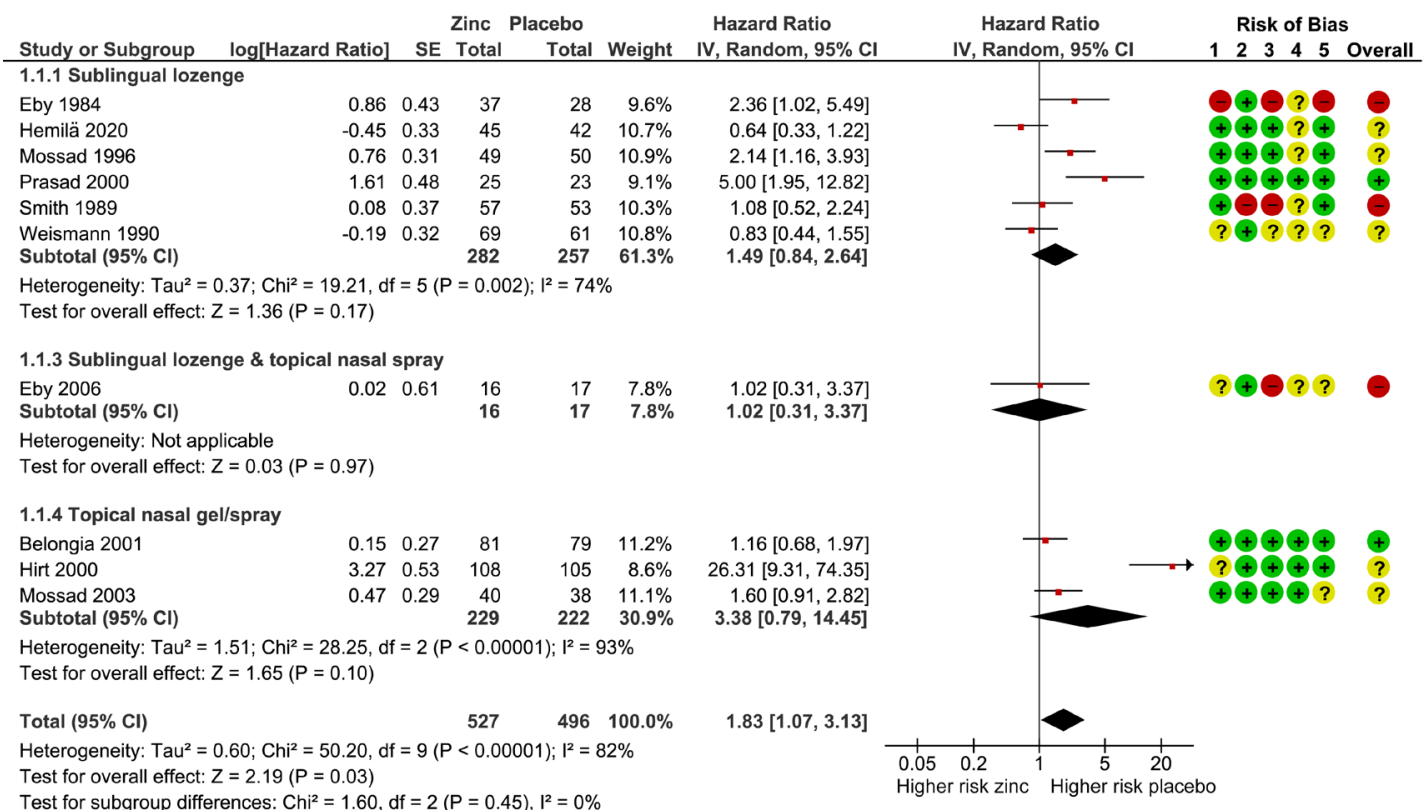

Risk of remaining symptomatic over 7-days: sublingual zinc compared to active control

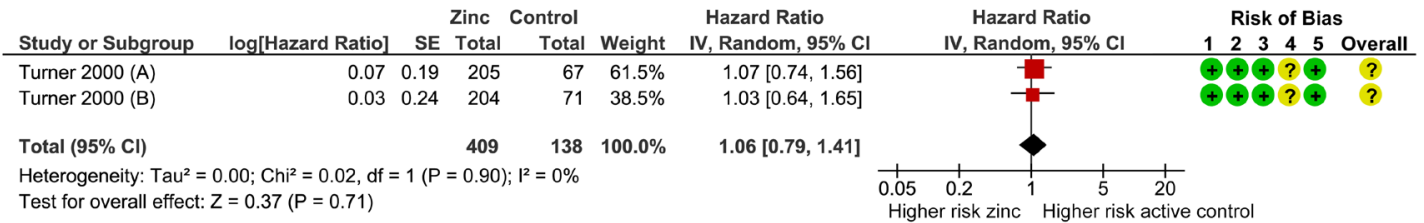

Mean days duration of symptoms: sublingual or topical nasal zinc compared to placebo

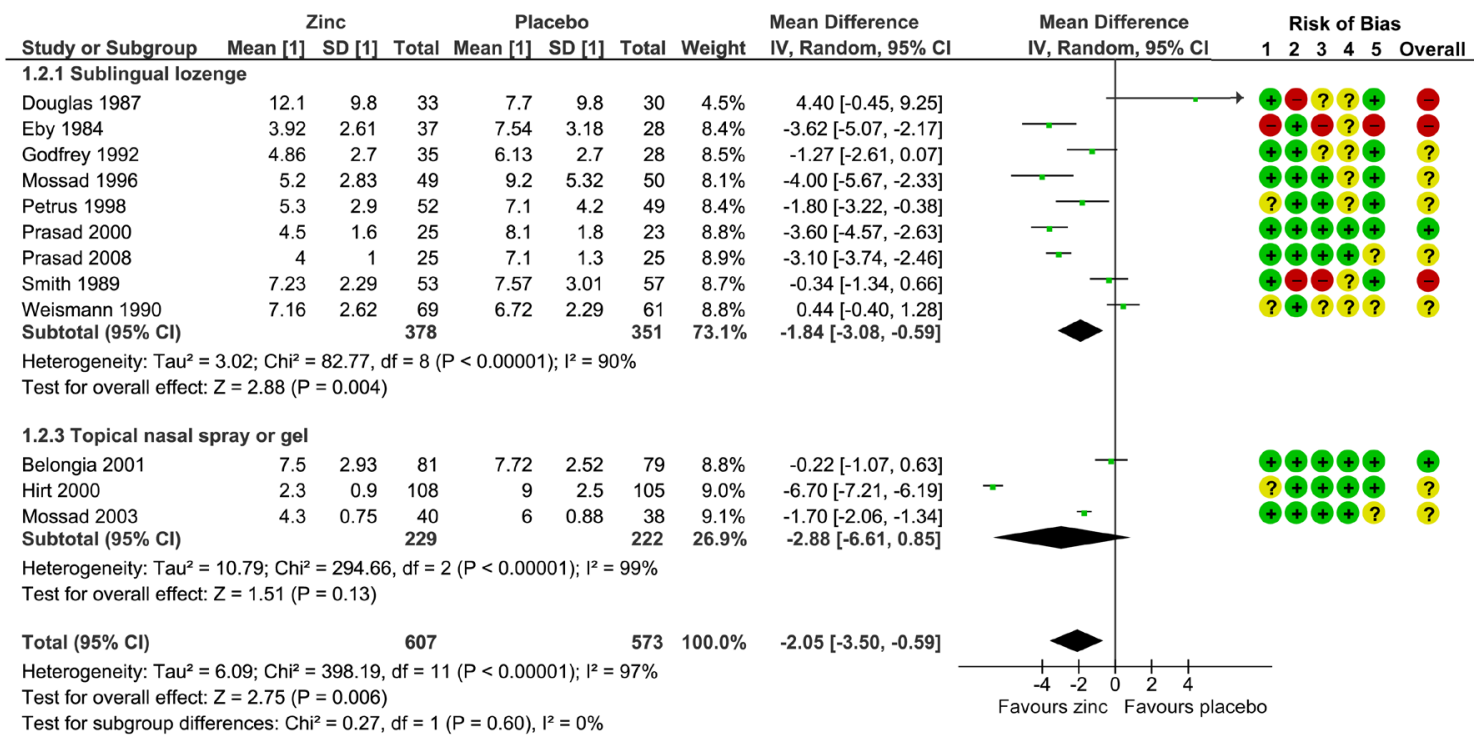

Mean days duration of symptoms: sublingual zinc compared to active control

\begin{tabular}{|c|c|c|c|c|c|c|c|c|c|c|c|}
\hline \multirow[b]{2}{*}{ Study or Subgroup } & \multicolumn{3}{|c|}{ Zinc } & \multicolumn{3}{|c|}{ Control } & \multicolumn{2}{|r|}{ Mean Difference } & \multirow{2}{*}{$\begin{array}{c}\text { Mean Difference } \\
\text { IV, Random, } 95 \% \mathrm{CI}\end{array}$} & \multicolumn{2}{|c|}{ Risk of Bias } \\
\hline & Mean [1] & SD [1] & Total & Mean [1] & SD [1] & Total & Weight & IV, Random, $95 \% \mathrm{CI}$ & & $\begin{array}{lllll}1 & 2 & 3 & 4 & 5\end{array}$ & Overall \\
\hline Turner 2000 (B) & 7.4096 & 3.8565 & 208 & 7.55 & 3.96 & 71 & $100.0 \%$ & $-0.14[-1.20,0.92]$ & & $\oplus+\oplus ?+$ & $?$ \\
\hline Total $(95 \% \mathrm{Cl})$ & & & 208 & & & 71 & $100.0 \%$ & $-0.14[-1.20,0.92]$ & & & \\
\hline $\begin{array}{l}\text { Heterogeneity: Not a } \\
\text { Test for overall effect }\end{array}$ & $\begin{array}{l}\text { licable } \\
Z=0.26(P\end{array}$ & $=0.80)$ & & & & & & & $\begin{array}{ccccc}-4 & -2 & 1 & 1 & 1 \\
-4 & 2 & 4 \\
\text { gvours zinc } & \text { Favours }\end{array}$ & ntrol & \\
\hline
\end{tabular}

Figure 4 Duration of illness. Risk of remaining symptomatic and mean days duration following treatment for communityacquired RTI or clinical colds from HRV inoculation. RoB-2 risk of bias legend: (1) randomisation process, (2) deviations from intended interventions, (3) missing outcome data, (4) measurement of the outcome, (5) selection of the reported result. HRV, human rhinovirus; RoB, risk of bias; RTI, respiratory tract infection. 
CI 0.04 to 0.38 ) compared with a $28 \%$ lower risk of developing milder symptoms (eg, common cold) (IRR 0.72, $95 \%$ CI 0.61 to 0.85 ) (moderate certainty/quality) ${ }^{76-78}$ However, due to more people contracting mild RTIs, the absolute risk reduction/risk difference was higher. Five mild RTIs were prevented per 100 person-months $(95 \%$ CI 2 to 7, NNT=20) compared with one moderate RTI per 100 person-months $(95 \%$ CI 1 to $2, \mathrm{NNT}=100$ ) (online supplemental appendix 4) ${ }^{76-78}$ Subgroup analysis found no significant differences according to age or zinc administration route or dose (online supplemental appendix $4)$.

\section{Human rhinovirus inoculation}

The effect of zinc lozenges compared with placebo for preventing RTIs caused by HRV inoculation was evaluated in two pre-exposure prevention (PrEP) RCTs with 53 participants, ${ }^{5860}$ and two post-exposure prevention (PEP) RCTs with 54 participants. ${ }^{59}$ Zinc had no effect on the risk of developing a clinical cold (RR 0.96, 95\% CI 0.77 to 1.21 , moderate certainty/quality) (figure 2). There were no significant differences between the effects of zinc compared with placebo for either the PrEP or PEP subgroup, or in the subgroup analysis comparing the two groups (figure 2). There were similar non-significant findings for the risks of developing a laboratory confirmed infection (online supplemental appendix 4).

\section{Adverse events}

No serious AEs were reported were reported in the four RCTs that used zinc for prevention (low certainty/ quality). ${ }^{75-78}$ Anosmia (loss of sense of smell) was not reported by the 1447 participants who used a zinc nasal spray nor the 1354 participants who used a placebo spray for 1 month (low certainty/quality). ${ }^{77} 78$ Compared with placebo, no differences in copper plasma concentration were found in the two smaller RCTs that evaluated $15 \mathrm{mg}$ of oral zinc for younger adults over 7 months ${ }^{75}$ or $45 \mathrm{mg}$ for older adults over 12 months ${ }^{76}$ (low certainty/quality). No differences in the of risk non-serious AEs from zinc compared with placebo controls were found (IRR 1.63, $95 \%$ CI 0.81 to 3.31 , low certainty/quality). ${ }^{757778}$

\section{Findings from treatment studies}

\section{Symptom severity}

Compared with placebo, a clinically important reduction of more than one point in the day 3 symptom severity scores was found for sublingual and topical nasal zinc (MD $-1.21,95 \%$ CI -1.74 to -0.66 , low certainty/quality) (figure 3) ${ }^{60-6365}$ In contrast, no differences in average daily symptom severity scores were found (SMD -0.15 , 95\% CI -0.43 to 0.13 , low certainty/quality) ${ }^{596064}$ Subgroup analyses found no significant differences according to zinc administration route or type of viral infection (online supplemental appendix 4).

\section{Symptom duration}

During the first week of illness, participants who used sublingual or topical nasal zinc were 1.8 times more likely to recover before those who used placebo (HR 1.83, 95\% CI 1.07 to 3.13 , low certainty/quality) (figure 4) ${ }^{61-63} 66-72$ An estimated 19 more adults per 100 were likely to remain symptomatic at the end of the first week if they used placebo rather than zinc (95\% CI 2 to $38, \mathrm{NNT}=5)$. Compared with placebo, zinc also reduced the mean duration of symptoms by 2 days (MD $-2.05,95 \%$ CI -3.50 to -0.59 , very low certainty/quality) (online supplemental appendix 4) ${ }^{61-65} 68-74$ Results from the subgroup analyses that compared zinc salts, administration routes and zinc lozenge doses were inconsistent (online supplemental appendix 4). There was low certainty/quality evidence that zinc lozenges were equivalent to an active control (HR $1.06,95 \%$ CI 0.79 to 1.41 ; MD -0.14 days, -1.20 to 0.92) (figure 4). ${ }^{51}$

\section{Adverse events}

No serious AEs were reported in the 19 RCTs that reported AEs (low certainty/quality). ${ }^{51} 59-7379$ However, the risk of any type of non-serious $\mathrm{AE}$ was higher from zinc use compared with placebo (RR 1.41, 95\% CI 1.17 to 1.69 ) (figure 5), with 14 more adults per 100 experiencing a nonserious $\mathrm{AE}$ ( $95 \%$ CI 9 to 20, $\mathrm{NNH}=7$, moderate certainty/ quality). ${ }^{5960626366-7173}$ Specifically, zinc increased the risk of nausea or gastrointestinal discomfort (RR 1.46, 95\% CI 1.03 to 2.06), ${ }^{59} 616365-68$ 71-74 mouth irritation or soreness from sublingual lozenges (RR 1.55, 95\% CI 1.05 to 2.29), ${ }^{59} 616365667273$ and taste aversion from sublingual lozenges (RR 2.11, 95\% CI 1.47 to 3.04), ${ }^{59} 6163656669717274$ but not nasal irritation or pain from topical nasal sprays or gels (RR 1.22, 95\% CI 0.72 to 2.05) (online supplemental appendix 4) ${ }^{606270}$ Zinc lozenges were more likely than nasal sprays and gels to cause any type of non-serious $\mathrm{AE}(\mathrm{p}=0.02)$ (online supplemental appendix 4). There was no difference in the rates of non-serious $\mathrm{AE}$ from zinc lozenges compared with active controls (RR 1.12, 95\% CI 0.76 to 1.65 ) (figure 5). ${ }^{5179}$

\section{DISCUSSION \\ Principal findings}

This rapid systematic review and meta-analysis advances the evidence on the effects of zinc for mild to moderate RTIs in adults without zinc deficiency. ${ }^{7-10} 398182$ However, despite additional searches through to August 2020, none of the RCTs were for the primary population of interest that was SARS-CoV-2 infection as the results from the registered clinical trials were all pending.

New evidence about zinc prophylaxis found that compared with placebo, zinc reduced the risk of developing symptoms consistent with a community-acquired viral RTI. The prophylactic effects were greatest for reducing the RR of developing more severe symptoms, such as fever and influenza-like illnesses. However, only four studies were identified, and none used laboratory tests to confirm a viral infection.

When zinc was used to treat symptoms consistent with mild to moderate viral RTIs, new evidence found that 
compared with placebo, there were clinically important reductions in day 3 symptom severity, but not average daily symptom severity scores. The difference in the severity results may reflect reporting bias and imprecision, as results could not be extracted for $13^{58} 5966-7479$ of the $20 \mathrm{RCTs}^{58-7479}$ that evaluated symptom severity, and there was only one overlapping $\mathrm{RCT}^{60}$ in the two meta-analyses conducted. Like previous reviews, ${ }^{7-10} 3981$ compared with placebo there were clinically important reductions in symptomatic duration from zinc use. However, there was also an increased risk of non-serious adverse effects that may reduce the tolerability or acceptability of some zinc formulations.

In contrast to these promising findings, following human rhinovirus inoculations, compared with placebo, sublingual zinc did not reduce the risk of developing an infection or symptoms of a clinical cold, nor were there any significant effects on symptom severity or duration of illness when zinc was compared with an active control. While the number of studies and sample sizes were small, it still raises the questions about the in vitro versus in vivo antiviral effects of zinc ions, at least against rhinoviruses, and comparative effectiveness.

\section{Strengths and weakness of the review}

Limitations to the certainty (quality) of the evidence included concerns about the RoB for most prevention, severity and duration outcomes, along with imprecision in the symptom severity lowering effects and inconsistencies in the treatment effect sizes for symptomatic duration. Further details about the impact of individual studies on GRADE certainty assessment of RoB, heterogeneity, imprecision and the overall quality of the evidence can be found in online supplemental appendix 5 .

The findings build on previous reviews. ${ }^{7-10} 398182$ Compared with two other systematic reviews conducted in the same period, ${ }^{10}{ }^{82}$ substantially more studies were identified in this review. This in part was due to searching non-English language databases and affirms calls to carefully consider which methods to restrict when conducting rapid reviews of traditional and complementary medicine. ${ }^{83}$ Other strengths included being the first systematic review of zinc for RTIs to synthesise hazard ratios for symptomatic duration, day 3 mean symptom severity scores around the peak of illness and risks of AEs. RoB appraisal at the outcome level rather than the study level helped optimise GRADE-certainty assessments that were both conducted following calibration exercises. Notwithstanding, there is always a degree of judgement that may vary between reviews. For instance, when appraising the available evidence for risk of serious AEs, we rated down one level for RoB and another for imprecision. However, it might also be reasonable to judge the RoB as not serious and the overall GRADE assessment as moderate, rather than low certainty/quality evidence. The assessments of publication bias were a potential limitation. While publication bias was not strongly suspected, visual inspection of funnel plots are necessarily subjective ${ }^{56}$ and a statistical test for hazard ratios was not performed.

Like other rapid reviews, single reviewers conducted many of the tasks that increases the risk of errors and inconsistencies. Nevertheless, we applied rigorous checks. For example, the use of a detailed data extraction form led to our reviewers being the first to notice that there were two 4 -arm RCTs ${ }^{51}$ in which the control lozenge contained an active ingredient, quinine hydrochloride that has broad-spectrum antiviral effects. ${ }^{84}$ Reclassifying them as an active control addressed unexplained inconsistencies identified by previous reviewers. ${ }^{39}$ Sensitivity analysis confirmed that the inclusion of these two RCTs, both with non-significant findings, did not substantially change the effect estimates for symptom duration (online supplemental appendix 4). We also determined that two earlier reviews ${ }^{9}{ }^{11}$ had incorrectly included the day 4 symptom severity scores from two RCTs ${ }^{6165}$ in their meta-analysis of average daily symptom severity. When these are removed, we found the effect of zinc was no longer significant.

Finally, while all protocol changes were declared, this increases the risk of selective reporting bias. Initiating the rapid review in the early stages of the COVID-19 pandemic necessitated rapid protocol development and registration. To help mitigate bias we sought blinded advice from our content experts and consumer/patient advocates and post hoc decisions were conservative with the rationale reported. Notably, while the published amendments ${ }^{34}$ to the protocol inclusion/exclusion criteria led to RCTs that inoculated participants with HRV also being included, the additional findings did not favour zinc. Post hoc decisions also ensured that the very serious indirectness of the available evidence was not overstated in the context of SARS-CoV-2 prevention or treatment.

\section{Is zinc more than a dietary supplement?}

The role of zinc in viral RTIs appears to extend beyond supplementing nutritional intake to prevent or treat zinc deficiency. ${ }^{85}$ The two RCTs that used prophylactic oral zinc excluded zinc deficiency prior to enrolment. ${ }^{75} 76$ While none of the other RCTs excluded deficiency, the risk was low as participants were generally healthy and the three RCTs conducted in China all used a low dose intranasal zinc spray $\left(1.15 \mathrm{mg}\right.$ daily ${ }^{77-79}$ that is unlikely to have substantial systemic effects. ${ }^{86}$ The rationale for topical intranasal and sublingual zinc is based on the in vitro effects of zinc ions that can inhibit viral replication, stabilise cell membranes and reduce mucosal inflammation. ${ }^{14}{ }^{86}$ However, other mechanisms may also be at play, at least for sublingual and oral administration as activation of T lymphocytes, monocytes and granulocytes has been observed in healthy young adult males within 24-48 hours of taking $15 \mathrm{mg}$ of oral zinc daily. ${ }^{87}$

\section{Implications for clinicians and consumers}

Zinc is readily available for consumers to self-prescribe. The marginal benefits, strain specificity, drug resistance and potential risks of other over-the-counter and 
prescription medications ${ }^{88-98}$ makes zinc a viable 'natural' alternative for the self-management of non-specific RTIs. It also provides clinicians with a management option for patients who are desperate for faster recovery times and might be seeking an unnecessary antibiotic prescription.

However, clinicians and consumers need to be aware that considerable uncertainty remains regarding the clinical efficacy of different zinc formulations, doses and administration routes, ${ }^{39678186}$ and the extent to which efficacy might be influenced by the ever changing epidemiology of the viruses that cause RTIs. The largest body of evidence comes from sublingual lozenges and zinc gluconate and acetate salts, suggesting these are suitable choices. Yet, this does not mean that other administration routes and zinc salts are less effective. The new evidence on the prophylactic effects of low dose nasal sprays ${ }^{778}$ adds weight to the otherwise inconclusive findings from the handful of RCTs evaluating zinc nasal sprays or gels for acute treatment. ${ }^{6266687079}$ A minimum therapeutic dose for zinc is also yet to be determined. An earlier review suggested the minimum dose for sublingual lozenges is $75 \mathrm{mg} .{ }^{8}$ However, our analysis does not support this conclusion. Further, a daily oral dose of $15 \mathrm{mg}$ has been shown to upregulate lymphocytes within days, ${ }^{8799}$ so it is plausible that much lower doses might also be effective.

The minimum time frame in which zinc should be started is also unclear. Most of the RCTs included in this review commenced zinc within 24 hours from the onset of symptoms and some guidelines have claimed that zinc 'only works if you start taking them within 24 hours' ${ }^{5}$ Yet, in the post hoc subgroup analyses, the duration of illness was also reduced in the subset of RCTs in which participants commenced zinc up to 3 days from the onset of symptoms. Further, in a preliminary analysis for one of the included RCTs, the investigators briefly report that the significant reduction in the duration of symptoms remained when participants with symptoms of up to 10 days duration were included in the analysis. ${ }^{63}$

Alongside potential benefits, consumers also seek detailed information about adverse effects and tolerability. Zinc was found to increase the risk of non-serious AEs. No serious AEs were reported, suggesting the risk is low. However, it cannot be ruled out as RCTs, especially those with small samples, are not well placed to identify rare events. If the rule of three is applied to determine maximum risk, ${ }^{100}$ then the upper $95 \%$ CI for a serious $\mathrm{AE}$ from prophylactic zinc would be 1.7/1000 person-months and for therapeutic zinc, 2.9/1000 participants. Indeed, postmarketing surveillance has identified cases of longlasting anosmia associated with a zinc gluconate nasal gel. ${ }^{101} 102$ Reassuringly, a loss of smell was not reported by any of the 1364 young adults who used nasal sprays for 1 month. Notwithstanding, anosmia is an early SARS-CoV-2 symptom, so any use of topical nasal zinc during the pandemic should be carefully considered and monitored.

Copper deficiency is another concern. Plasma copper levels and other laboratory parameters were stable following $15 \mathrm{mg}$ and $45 \mathrm{mg}$ for 7 months and 12 months, respectively. ${ }^{75}{ }^{76}$ However, contamination of the zinc intervention was found in one RCT, ${ }^{75}$ both RCTs were small and may be underpowered to detect a difference, only a single marker of copper status was measured, ${ }^{103}$ and intestinal absorption of zinc is influenced by a variety of factors including diet, medications, chronic diseases and increasing age. ${ }^{1517}$

\section{Implications for research}

Given the limited therapeutic options for preventing and treating viral RTIs, further research is indicated to better understand zinc's mechanisms of action, the optimum administration routes, formulations and dose, the minimum time-frame in which zinc should be started following an acute infection and the duration of therapy.

Except for one RCT that evaluated the effects of zinc on cognitive function, ${ }^{58} 80$ the symptomatic and functional impact on the participants' QoL was not assessed. These outcomes are important to patients. Questionnaires like the Wisconsin Upper Respiratory Symptom Survey-24 that assess both symptom severity and QoL are therefore recommended. ${ }^{104}$

The review findings align with calls for more immunonutrition research, particularly in populations with a higher SARS-CoV-2 risk. ${ }^{17105}$ Results from seven RCTs evaluating various zinc doses, salts and administration routes for the prevention or treatment of SARS-CoV-2 are all pending. These RCTs will continue to be tracked and the review periodically updated and reported until there is moderate certainty/quality in the evidence, or no results are pending. However, based on the limited information reported in the protocols, some of the choices for zinc interventions appear to be arbitrary. Future SARS-CoV-2 clinical trials should consider replicating the RCTs with positive results for other viral RTIs and consider focusing on high-risk groups. Trials also need to determine if zinc requires a carrier or an ionophore, such as hydroxychloroquine, ${ }^{28}$ and compare the risks and benefits. According to our review findings and preliminary in vitro SARS-CoV research, ${ }^{12}$ it is plausible that zinc may be effective when used on its own.

\section{CONCLUSIONS}

In adult populations in which zinc deficiency is unlikely, our review found when zinc was used for prophylaxis, there was a lower risk of contracting a clinical illness consistent with a community-acquired viral RTI, but not following direct HRV inoculation. When used for treatment, zinc was found to shorten the duration of symptoms and reduce day 3 symptomatic severity, but not overall daily symptom severity. While there was an increased risk of non-serious AEs that may limit tolerability for some, the risk of serious AEs was low. Limitations to the GRADE certainty/quality assessments of the available evidence included a high RoB and/or small sample sizes in primary studies, and considerable heterogeneity in the duration effect estimates. We were unable to answer 
questions about the comparative efficacy, effectiveness and acceptability of different zinc formulations and doses, and their mechanisms of action. Prior to recommending zinc, patient preferences, financial and opportunity costs, and availability of different zinc interventions should be considered. Clarification of the efficacy and mechanism of zinc in viral respiratory infections, including SARSCoV-2 infections, warrants further research.

\section{Author affiliations}

${ }^{1}$ NICM Health Research Institute, Western Sydney University, Penrith, New South Wales, Australia

${ }^{2}$ Helfgott Research Institute, National University of Natural Medicine, Portland, Oregon, USA

${ }^{3}$ Seattle, Washington, USA

${ }^{4}$ National Centre for Naturopathic Medicine, Southern Cross University, Lismore, New South Wales, Australia

${ }^{5}$ Division of Infectious Diseases, Department of Medicine, Health Sciences, McMaster University, Hamilton, Ontario, Canada

${ }^{6}$ The Menzies Centre for Health Policy, The University of Sydney, Sydney, New South Wales, Australia

Twitter Jennifer Hunter Western Sydney University @westernsydneyu and Susan Arentz @sarentz

Contributors All authors met the criteria of authorship as outlined in the ICMJE and approved this manuscript for submission. $\mathrm{JH}$ and SA contributed equally to this work. Specific contributions were as follows: conceptualisation: SA, JH and JG; methodology: JH, SA, JG, JB, GY, DM and SL; project administration: SA and JH; investigation: JH, SA, JG, JB and GY; data curation: $\mathrm{SA}$ and $\mathrm{JH}$; formal analysis: $\mathrm{JH}$, $\mathrm{SA}$, JB and SPM; validation: $\mathrm{SA}, \mathrm{JH}$ and GY; visualisation: $\mathrm{JH}$ and $\mathrm{SA}$; writing original draft: JH, SA, JB and SL; writing, review and editing: JH, SA, JG, JB, GY, SPM, DM and SL.

Funding The authors have not declared a specific grant for this research from any funding agency in the public, commercial or not-for-profit sectors.

Competing interests All authors have completed the Unified Competing Interest form (available on request from the corresponding author) and declare: no support from any organisation for the submitted work. DM and SL have no financial relationships with any organisations that might have an interest in the submitted work in the previous 3 years and no other relationships or activities that could appear to have influenced the submitted work. SA, JH and GY are academic researchers at NICM Health Research Institute. As a medical research institute, NICM Health Research Institute receives research grants and donations from foundations, universities, government agencies, individuals and industry. Sponsors and donors provide untied funding for work to advance the vision and mission of the Institute. This review was not undertaken as part of a contractual relationship with any donor or sponsor. JH is an academic general practitioner with a clinical interest in integrative medicine, has received payment for providing expert advice about traditional, complementary and integrative medicine, including nutraceuticals, to industry, government bodies and non-government organisations, and spoken at workshops, seminars and conferences for which registration, travel and/or accommodation has been paid for by the organisers. SA is a naturopathic practitioner at an obstetrics and gynaecology clinic in Sydney, Australia. She has received payment for providing expert editing of naturopathic and herbal medicine educational programmes, and for investigation of naturopathy, herbal medicines and nutraceuticals in clinical trials and spoken at workshops, seminars and conferences for which registration, travel and/or accommodation has been paid by the organisers. GY is an academic researcher with interest in complementary and integrative medicine. She has spoken at research workshops, seminars and conferences for which registration and travel has been paid by the organisers. JG is a naturopathic doctor and director of a functional bowel disease clinic in Colorado Springs, USA. JG has spoken at research conferences for which registration, travel and/or accommodation has been paid by the organisers. He is also a research investigator at the Helfgott Research Institute, National University of Natural Medicine. As a medical research institute, Helfgott receives research grants and untied donations from foundations, universities, government agencies, individuals, and industry. This review was not undertaken as part of a contractual relationship with any donor or sponsor. JB is an independent librarian and reports personal fees from Helfgott Research Institute, National University of Natural Medicine, during the conduct of the study. SPM is an academic researcher at Southern Cross University where he is a Professor of Traditional, Complementary and Integrative Medicine. SPM has received payment for providing expert advice to industry, government bodies and non-government organisations, and spoken at workshops, seminars and conferences for which registration, travel and/or accommodation has been paid for by the organisers. This review was not undertaken as part of a contractual relationship with any donor or sponsor.

Patient consent for publication Not applicable.

Provenance and peer review Not commissioned; externally peer reviewed.

Data availability statement Data are available on reasonable request. Additional data may be made available upon request.

Supplemental material This content has been supplied by the author(s). It has not been vetted by BMJ Publishing Group Limited (BMJ) and may not have been peer-reviewed. Any opinions or recommendations discussed are solely those of the author(s) and are not endorsed by BMJ. BMJ disclaims all liability and responsibility arising from any reliance placed on the content. Where the content includes any translated material, BMJ does not warrant the accuracy and reliability of the translations (including but not limited to local regulations, clinical guidelines, terminology, drug names and drug dosages), and is not responsible for any error and/or omissions arising from translation and adaptation or otherwise.

Open access This is an open access article distributed in accordance with the Creative Commons Attribution Non Commercial (CC BY-NC 4.0) license, which permits others to distribute, remix, adapt, build upon this work non-commercially, and license their derivative works on different terms, provided the original work is properly cited, appropriate credit is given, any changes made indicated, and the use is non-commercial. See: http://creativecommons.org/licenses/by-nc/4.0/.

\section{ORCID iDs}

Jennifer Hunter http://orcid.org/0000-0002-6109-9134

Susan Arentz http://orcid.org/0000-0002-6721-472X

Joshua Goldenberg http://orcid.org/0000-0003-2572-3929

Guoyan Yang http://orcid.org/0000-0002-8012-2379

Jennifer Beardsley http://orcid.org/0000-0002-7848-5381

Stephen P Myers http://orcid.org/0000-0002-4510-9945

Dominik Mertz http://orcid.org/0000-0003-4337-1613

Stephen Leeder http://orcid.org/0000-0003-3532-7137

\section{REFERENCES}

1 Avendaño Carvajal L, Perret Pérez C. Epidemiology of respiratory infections. Pediatric Respiratory Diseases 2020:263-72.

2 Eccles R, Weber O. Common cold. Secaucus: Birkhäuser, 2009.

$3 \mathrm{CDC}$. Antibiotic prescribing and use in doctor's offices: adults treatment recommendations, 2017. Centres for disease control and prevention. Available: https://www.cdc.gov/antibiotic-use/index. html [Accessed 4 Oct 2020].

4 Gruffydd-Jones K, Hickman K. Managing dilemmas in respiratory infections and antibiotic prescribing. Primary Care Respiratory update 2018;5:32-5 https://www.pcrs-uk.org/sites/pcrs-uk.org/files/ RTI Antbx 5 12018.pdf

5 DeGeorge KC, Ring DJ, Dalrymple SN. Treatment of the common cold. Am Fam Physician 2019;100:281-9.

6 Jackson JL, Lesho E, Peterson C. Zinc and the common cold: a meta-analysis revisited. J Nutr 2000;130:1512s-5.

7 D'Cruze H, Arroll B, Kenealy T. Is intranasal zinc effective and safe for the common cold? a systematic review and meta-analysis. $J$ Prim Health Care 2009;1:134-9.

8 Hemilä $\mathrm{H}$. Zinc lozenges may shorten the duration of colds: a systematic review. Open Respir Med J 2011;5:51.

9 Science M, Johnstone J, Roth DE, et al. Zinc for the treatment of the common cold: a systematic review and meta-analysis of randomized controlled trials. CMAJ 2012;184:E551-61.

10 Wang MX, Win SS, Pang J. Zinc supplementation reduces common cold duration among healthy adults: a systematic review of randomized controlled trials with micronutrients supplementation. Am J Trop Med Hyg 2020;103:86-99.

11 Singh M, Das RR. Withdrawn: zinc for the common cold. Cochrane Database Syst Rev 2015;4:Cd001364.

12 te Velthuis AJW, van den Worm SHE, Sims AC, et al. Zn2+ inhibits coronavirus and arterivirus RNA polymerase activity in vitro and zinc ionophores block the replication of these viruses in cell culture. PLoS Pathog 2020;6:e1001176. 
13 Skalny AV, Rink L, Ajsuvakova OP, et al. Zinc and respiratory tract infections: perspectives for COVID-19 (review). Int J Mol Med 2020;46:17-26.

14 Hulisz D. Efficacy of zinc against common cold viruses: an overview. J Am Pharm Assoc 2004;44:594-603.

15 Arentz S, Hunter J, Yang G, Goldenberg J, et al. Zinc for the prevention and treatment of SARS-CoV-2 and other acute viral respiratory infections: a rapid review. Adv Integr Med 2020;7:252-60.

16 Ischia J, Bolton DM, Patel O. Why is it worth testing the ability of zinc to protect against ischaemia reperfusion injury for human application. Metallomics 2019;11:1330-43.

17 Mossink JP. Zinc as nutritional intervention and prevention measure for COVID-19 disease. BMJ nutr prev health 2020:bmjnph2020-000095.

18 O'Connor A. Taking supplements probably won't help, and may harm, 2020. The New York Times. Available: https://www.nytimes. com/2020/03/23/well/live/coronavirus-supplements-herbs-vitaminscolds-flu.html

19 Ahmed I, Hasan M, Akter R, et al. Behavioral preventive measures and the use of medicines and herbal products among the public in response to Covid-19 in Bangladesh: a cross-sectional study. PLOS One 2020:15:2020.08.15.20175513.

20 Khurana A, Kaushal GP, gupta R. Prevalence and clinical correlates of COVID-19 outbreak among healthcare workers in a tertiary level Hospital. medRxiv 2020:2020.07.21.20159301.

21 Alam MM, Mahmud S, Rahman MM, et al. Clinical outcomes of early treatment with doxycycline for 89 high-risk COVID-19 patients in long-term care facilities in New York. Cureus 2020;12:e9658.

22 Bahloul M, Ketata W, Lahyeni D, et al. Pulmonary capillary leak syndrome following COVID-19 virus infection. J Med Virol 2021;93:94-96

23 Derwand R, Scholz M, Zelenko V. COVID-19 outpatients: early riskstratified treatment with zinc plus low-dose hydroxychloroquine and azithromycin: a retrospective case series study. Int J Antimicrob Agents 2020;56:106214.

24 Enzmann MO, Erickson MP, Grindeland CJ, et al. Treatment and preliminary outcomes of 150 acute care patients with COVID-19 in a rural health system in the Dakotas. Epidemiol Infect 2020;148:e124.

25 Kang JE, Rhie SJ. Practice considerations on the use of investigational anti-COVID-19 medications: dosage, administration and monitoring. J Clin Pharm Ther 2020;45:1199-205.

26 Sattar Y, Connerney M, Rauf H, et al. Three cases of COVID-19 disease with colonic manifestations. Am J Gastroenterol 2020;115:948-50.

27 Shady A, Singh AP, Gbaje E, et al. Characterization of patients with COVID-19 admitted to a community hospital of East Harlem in New York City. Cureus 2020;12:e9836.

28 Rahimian JO, Yaghi S, Liu M. Treatment with zinc is associated with reduced in-hospital mortality among COVID-19 patients: a multicenter cohort study, 2020

29 Carlucci PM, Ahuja T, Petrilli C, et al. Zinc sulfate in combination with a zinc ionophore may improve outcomes in hospitalized COVID-19 patients. J Med Microbiol 2020;69:1228-34.

30 Capone S, Abramyan S, Ross B, et al. Characterization of critically ill COVID-19 patients at a brooklyn safety-net hospital. Cureus 2020;12:e9809.

31 Yao JS, Paguio JA, Dee EC, et al. The minimal effect of zinc on the survival of hospitalized patients with COVID-19: an observational study. Chest 2021;159:108-11.

32 Steel A, Wardle J, Lloyd I. The potential contribution of traditional, complementary and integrative treatments in acute viral respiratory tract infections: rapid reviews in response to the COVID-19 pandemic. Adv Integr Med 2020;7:181-2.

33 Arentz S, Hunter J, Goldenberg J. Protocol for a rapid review of zinc for the prevention or treatment of COVID-19 and other coronavirusrelated respiratory tract infections in humans. prospero 2020 CRD42020182044: National Institute for health research 2020. Available: www.crd.york.ac.uk/prospero/display record.php? ID=CRD42020182044

34 Hunter J, Arentz S, Goldenberg J, et al. Rapid review protocol: zinc for the prevention or treatment of COVID-19 and other coronavirusrelated respiratory tract infections. Integr Med Res 2020;9:100457.

35 Garritty C, Gartlehner G, Kamel C. Cochrane rapid reviews. interim guidance from the cochrane rapid reviews methods group, 2020. https://methods.cochrane.org/rapidreviews/sites/methods. cochrane.org.rapidreviews/files/public/uploads/cochrane_rr guidance-23mar2020-final.pdf

36 Higgins JPT TJ, Chandler J, Cumpston M. Cochrane Handbook for systematic reviews of interventions. John Wiley \& Sons, 2019.
37 Page MJ, McKenzie JE, Bossuyt PM, et al. The prisma 2020 statement: an updated guideline for reporting systematic reviews. BMJ 2021;372:n71.

38 Sterne JAC, Savović J, Page MJ, et al. Rob 2: a revised tool for assessing risk of bias in randomised trials. BMJ 2019;366:14898

39 Hemilä H. Zinc lozenges and the common cold: a meta-analysis comparing zinc acetate and zinc gluconate, and the role of zinc dosage. JRSM Open 2017;8:2054270417694291.

40 Rohatgi A. Webplotdigitizer version 4.2, 2019. Available: https:// automeris.io/WebPlotDigitizer

41 The Cochrane Collaboration. Review manager (revman) [program] 5.3 version. Copenhagen: The Nordic Cochrane Centre, 2014.

42 R_core_team. R: a language and environment for statistical computing Vienna, Austria, 2019. Available: https://www.R-project. org/

43 Balduzzi S, Rücker G, Schwarzer G. How to perform a metaanalysis with R: a practical tutorial. Evid Based Ment Health 2019:22:153-60.

44 Evidence_Prime I. Gradepro guideline development tool [software], 2020. Mcmaster University. Available: gradepro.org

45 Thorlund K, Walter SD, Johnston BC, et al. Pooling health-related quality of life outcomes in meta-analysis-a tutorial and review of methods for enhancing interpretability. Res Synth Methods 2011;2:188-203.

46 Jackson GG, Dowling HF, Spiesman IG, et al. Transmission of the common cold to volunteers under controlled conditions. I. the common cold as a clinical entity. AMA Arch Intern Med 1958:101:267-78

47 Tierney JF, Stewart LA, Ghersi D. Practical methods for incorporating summary time-to-event data into meta-analysis. additional file 1: HR calculations spreadsheet. Trials 2007;8 https://static-content.springer.com/esm/art\%3A10.1186\% 2F1745-6215-8-16/MediaObjects/13063_2006_188_MOESM1_ ESM.xls

48 Sun X, loannidis JPA, Agoritsas T, et al. How to use a subgroup analysis: users' guide to the medical literature. JAMA 2014;311:405-11.

49 European Commission Health, Consumer Protection DirectorateGeneral: Scientific Committee on Food. Opinion of the scientific committee on food on the tolerable upper intake level of zinc expressed on 5 March 2003, 2003.

50 Cohen J. Statistical power analysis for the behavioral sciences. Academic press, 2013

51 Turner RB, Cetnarowski WE. Effect of treatment with zinc gluconate or zinc acetate on experimental and natural colds. Clin Infect Dis 2000;31:1202-8.

52 Norman GR, Sloan JA, Wyrwich KW. Interpretation of changes in health-related quality of life: the remarkable universality of half a standard deviation. Med Care 2003:41:582-92.

53 Gwaltney JM, Hendley JO, Simon G, et al. Rhinovirus infections in an industrial population. II. characteristics of illness and antibody response. JAMA 1967:202:494-500.

54 The GRADE Working Group. Handbook for grading the quality of evidence and the strength of recommendations using the grade approach, 2013.

55 Sterne JAC, Sutton AJ, loannidis JPA, et al. Recommendations for examining and interpreting funnel plot asymmetry in meta-analyses of randomised controlled trials. BMJ 2011;343:d4002.

56 Debray TPA, Moons KGM, Riley RD. Detecting small-study effects and funnel plot asymmetry in meta-analysis of survival data: a comparison of new and existing tests. Res Synth Methods 2018:9:41-50.

57 Silk R, LeFante C. Safety of zinc gluconate glycine (cold-eeze) in a geriatric population: a randomized, placebo-controlled, doubleblind trial. Am J Ther 2005;12:612-7.

58 Al-Nakib W, Higgins PG, Barrow I, et al. Prophylaxis and treatment of rhinovirus colds with zinc gluconate lozenges. J Antimicrob Chemother 1987;20:893-901.

59 Farr BM, Conner EM, Betts RF, et al. Two randomized controlled trials of zinc gluconate lozenge therapy of experimentally induced rhinovirus colds. Antimicrob Agents Chemother 1987;31:1183-7.

60 Turner RB. Ineffectiveness of intranasal zinc gluconate for prevention of experimental rhinovirus colds. Clin Infect Dis 2001;33:1865-70.

61 Prasad AS, Fitzgerald JT, Bao B, et al. Duration of symptoms and plasma cytokine levels in patients with the common cold treated with zinc acetate. A randomized, double-blind, placebo-controlled trial. Ann Intern Med 2000;133:245-16.

62 Belongia EA, Berg R, Liu K. A randomized trial of zinc nasal spray for the treatment of upper respiratory illness in adults. Am J Med 2001:111:103-8. 
63 Eby GA, Davis DR, Halcomb WW. Reduction in duration of common colds by zinc gluconate lozenges in a double-blind study. Antimicrob Agents Chemother 1984;25:20-4.

64 Petrus EJ, Lawson KA, Bucci LR, et al. Randomized, doublemasked, placebo-controlled clinical study of the effectiveness of zinc acetate lozenges on common cold symptoms in allergy-tested subjects. Curr Ther Res Clin Exp 1998;59:595-607.

65 Prasad AS, Beck FWJ, Bao B, et al. Duration and severity of symptoms and levels of plasma interleukin-1 receptor antagonist, soluble tumor necrosis factor receptor, and adhesion molecules in patients with common cold treated with zinc acetate. $J$ Infect Dis 2008;197:795-802

66 Eby GA, Halcomb WW. Ineffectiveness of zinc gluconate nasal spray and zinc orotate lozenges in common-cold treatment: a double-blind, placebo-controlled clinical trial. Altern Ther Health Med 2006;12:34-8.

67 Hemilä H, Haukka J, Alho M, et al. Zinc acetate lozenges for the treatment of the common cold: a randomised controlled trial. BMJ Open 2020;10:e031662.

68 Hirt M, Nobel S, Barron E. Zinc nasal gel for the treatment of common cold symptoms: a double-blind, placebo-controlled trial. Ear Nose Throat J 2000;79:778-82.

69 Weismann K, Jakobsen JP, Weismann JE, et al. Zinc gluconate lozenges for common cold. a double-blind clinical trial. Dan Med Bull 1990;37:279-81.

70 Mossad SB. Effect of zincum gluconicum nasal gel on the duration and symptom severity of the common cold in otherwise healthy adults. QJM 2003;96:35-43.

71 Mossad SB, Macknin ML, Medendorp SV, et al. Zinc gluconate lozenges for treating the common cold. A randomized, doubleblind, placebo-controlled study. Ann Intern Med 1996;125:81-8.

72 Smith DS, Helzner EC, Nuttall CE, et al. Failure of zinc gluconate in treatment of acute upper respiratory tract infections. Antimicrob Agents Chemother 1989;33:646-8.

73 Godfrey JC, Conant Sloane B, Smith DS, et al. Zinc gluconate and the common cold: a controlled clinical study. $J$ Int Med Res 1992;20:234-46.

74 Douglas RM, Miles HB, Moore BW, et al. Failure of effervescent zinc acetate lozenges to alter the course of upper respiratory tract infections in Australian adults. Antimicrob Agents Chemother 1987;31:1263-5.

75 Veverka DV, Wilson C, Martinez MA, et al. Use of zinc supplements to reduce upper respiratory infections in United States air force academy cadets. Complement Ther Clin Pract 2009;15:91-5.

76 Prasad AS, Beck FWJ, Bao B, et al. Zinc supplementation decreases incidence of infections in the elderly: effect of zinc on generation of cytokines and oxidative stress. Am J Clin Nutr 2007;85:837-44.

77 Wei J, Chen HW, You LH. Zinc gluconate nasal spray for the prevention of upper respiratory tract infection: A randomised, double-blinded, placebo-controlled trial]. Med J Chinese Liber Army 2009;34:838-40 http://www.plamj.org/index.php/plamj

78 Zhang LJ, Liu GX, Zhang YX. Zinc gluconate nasal spray for the prevention of acute upper respiratory tract infection]. J Preven Med Infor 2009;25:508-10.

79 Yao WZ, Yang W, Shen N. Zinc gluconate nasal spray versus common cold nasal spray in treating common cold: A randomised, multi-center, controlled trial]. Chinese Journal of Clinical Pharmacology 2005;21:87-90.

80 Smith AP, Tyrrell DA, Al-Nakib W, et al. Effects of zinc gluconate and nedocromil sodium on performance deficits produced by the common cold. J Psychopharmacol 1991;5:251-4.

81 Hemilä $\mathrm{H}$, Chalker $\mathrm{E}$. The effectiveness of high dose zinc acetate lozenges on various common cold symptoms: a meta-analysis. BMC Fam Pract 2015;16:24.

82 Abioye Al, Bromage S, Fawzi W. Effect of micronutrient supplements on influenza and other respiratory tract infections among adults: a systematic review and meta-analysis. BMJ Glob Health 2021;6:e003176.

83 Hunter J, Arentz S, Goldenberg J. Choose your shortcuts wisely: COVID-19 rapid reviews of traditional, complementary and integrative medicine. Integrative Medicine Research2020;100484.

84 Ahidjo BA, Loe MWC, Ng YL, et al. Current perspective of antiviral strategies against COVID-19. ACS Infect Dis 2020;6:1624-34.

85 Lassi ZS, Moin A, Bhutta ZA. Zinc supplementation for the prevention of pneumonia in children aged 2 months to 59 months. Cochrane Database Syst Rev 2016;12:Cd005978.

86 Eby GA. Zinc lozenges as cure for the common cold--a review and hypothesis. Med Hypotheses 2010;74:482-92.

87 Aydemir TB, Blanchard RK, Cousins RJ. Zinc supplementation of young men alters metallothionein, zinc transporter, and cytokine gene expression in leukocyte populations. Proc Natl Acad Sci U S A 2006;103:1699-704.

88 Heneghan CJ, Onakpoya I, Jones MA, et al. Neuraminidase inhibitors for influenza: a systematic review and meta-analysis of regulatory and mortality data. Health Technol Assess 2016;20:1-242.

89 Hussain M, Galvin HD, Haw TY, et al. Drug resistance in influenza a virus: the epidemiology and management. Infect Drug Resist 2017; $10: 121-34$.

90 De Sutter AI, Saraswat A, van Driel ML. Antihistamines for the common cold. Cochrane Database Syst Rev 2015;11:Cd009345.

91 Deckx L, De Sutter Al, Guo L, et al. Nasal decongestants in monotherapy for the common cold. Cochrane Database Syst Rev 2016;10:Cd009612.

92 Hao Q, Dong BR, Wu T. Probiotics for preventing acute upper respiratory tract infections. Cochrane Database Syst Rev 2015;2:Cd006895.

93 Hawkins J, Baker C, Cherry L, et al. Black elderberry (sambucus nigra) supplementation effectively treats upper respiratory symptoms: a meta-analysis of randomized, controlled clinical trials. Complement Ther Med 2019;42:361-5.

94 Hayward G, Thompson MJ, Perera R, et al. Corticosteroids for the common cold. Cochrane Database Syst Rev 2015;10:Cd008116.

95 Hemilä H, Chalker E. Vitamin C for preventing and treating the common cold. Cochrane Database Syst Rev 2013;1:Cd000980.

96 Hsu J, Santesso N, Mustafa R, et al. Antivirals for treatment of influenza: a systematic review and meta-analysis of observational studies. Ann Intern Med 2012;156:512-24.

97 Karsch-Völk M, Barrett B, Kiefer D, et al. Echinacea for preventing and treating the common cold. Cochrane Database Syst Rev 2014;2:Cd000530.

98 Taverner D, Latte J. Nasal decongestants for the common cold. Cochrane Database Syst Rev 2007:Cd001953.

99 Aydemir TB, Liuzzi JP, McClellan S, et al. Zinc transporter zip8 (SLC39A8) and zinc influence IFN-gamma expression in activated human T cells. J Leukoc Biol 2009;86:337-48.

100 Eypasch E, Lefering R, Kum CK, et al. Probability of adverse events that have not yet occurred: a statistical reminder. $B M J$ 1995;311:619-20.

101 Jafek BW, Linschoten MR, Murrow BW. Anosmia after intranasal zinc gluconate use. Am J Rhinol 2004;18:137-41.

102 Alexander TH, Davidson TM, Zinc I. Intranasal zinc and anosmia: the zinc-induced anosmia syndrome. Laryngoscope 2006;116:217-20.

103 Duncan A, Yacoubian C, Watson N, et al. The risk of copper deficiency in patients prescribed zinc supplements. J Clin Pathol 2015;68:723-5.

104 Department of family medicine and community health. Wisconsin upper respiratory symptom survey (WURSS), 2020. Available: https://www.fammed.wisc.edu/wurss/ [Accessed 12 Aug 2020].

105 Derbyshire E, Delange J. COVID-19: is there a role for immunonutrition, particularly in the over 65s? BMJ Nutr Prev Health 2020;3:100-5. 